Revue des patrimoines

46 | 2022

Le patrimoine de la Justice

\title{
Une prison modèle ? La prison circulaire d'Autun, un projet idéal à l'épreuve de la réalité
}

A model prison? The circular prison of Autun, an ideal project to the test of reality

\section{Agathe Mathiaut-Legros}

\section{CpenEdition}

\section{Journals}

Édition électronique

URL : https://journals.openedition.org/insitu/33830

DOI : $10.4000 /$ insitu. 33830

ISSN : $1630-7305$

Éditeur

Ministère de la Culture

Référence électronique

Agathe Mathiaut-Legros, « Une prison modèle ? La prison circulaire d'Autun, un projet idéal à l'épreuve de la réalité », In Situ [En ligne], 46 | 2022, mis en ligne le 18 janvier 2022, consulté le 15 février 2023. URL : http://journals.openedition.org/insitu/33830 ; DOI : https://doi.org/10.4000/insitu.33830

Ce document a été généré automatiquement le 3 février 2022

\section{(†) $९$}

Creative Commons - Attribution - Pas d'Utilisation Commerciale - Pas de Modification 4.0 International - CC BY-NC-ND 4.0

https://creativecommons.org/licenses/by-nc-nd/4.0/ 


\title{
Une prison modèle ? La prison circulaire d'Autun, un projet idéal à l'épreuve de la réalité
}

\author{
A model prison? The circular prison of Autun, an ideal project to the test of \\ reality
}

Agathe Mathiaut-Legros

1 L'ancienne prison d'Autun, utilisée comme telle de 1856 jusqu'aux années 1950 et quasiment inoccupée depuis, est remarquable à plus d'un titre. Rare exemple d'édifice panoptique de plan circulaire construit en France, elle traduit avec une grande fidélité le modèle du panopticon de Jeremy Bentham. Quelques particularités semblent être le fait de son architecte, André Berthier (1811-1873), mais poursuivent si bien le concept initial qu'elles dénotent une véritable appropriation du principe panoptique. Si l'état actuel de conservation du bâtiment est globalement médiocre, il n'accuse cependant que les dégâts provoqués par le temps et la négligence humaine. Pour de multiples raisons, l'édifice n'a jamais jusqu'à ce jour suscité suffisamment d'intérêt pour faire l'objet de profondes modifications. Classé au titre des monuments historiques en $2017^{2}$, il est aujourd'hui intégré à un projet muséal qui devrait le conduire pour la première fois de son histoire à une véritable mutation d'usage.

2 Le musée Rolin, mitoyen, accueille depuis le xix ${ }^{e}$ siècle de riches collections d'art et d'archéologie, étroitement liées aux 2000 ans de l'histoire peu commune d'Autun, depuis sa fondation par Auguste au tournant de notre ère jusqu'au $\mathrm{xx}^{\mathrm{e}}$ siècle. Un ambitieux projet d'extension vise à étendre ce musée sur le bâtiment de la prison circulaire et sur celui du palais de justice mitoyen [fig. 1]. La prison deviendrait ainsi le centre d'un complexe muséal bordant toute la place Saint-Louis et faisant face à la cathédrale Saint-Lazare. Après plusieurs années d'études et de programmation, à l'issue d'un concours d'architecture, une équipe de maîtrise d'œuvre a été retenue début 2020 pour donner forme à ce projet. 


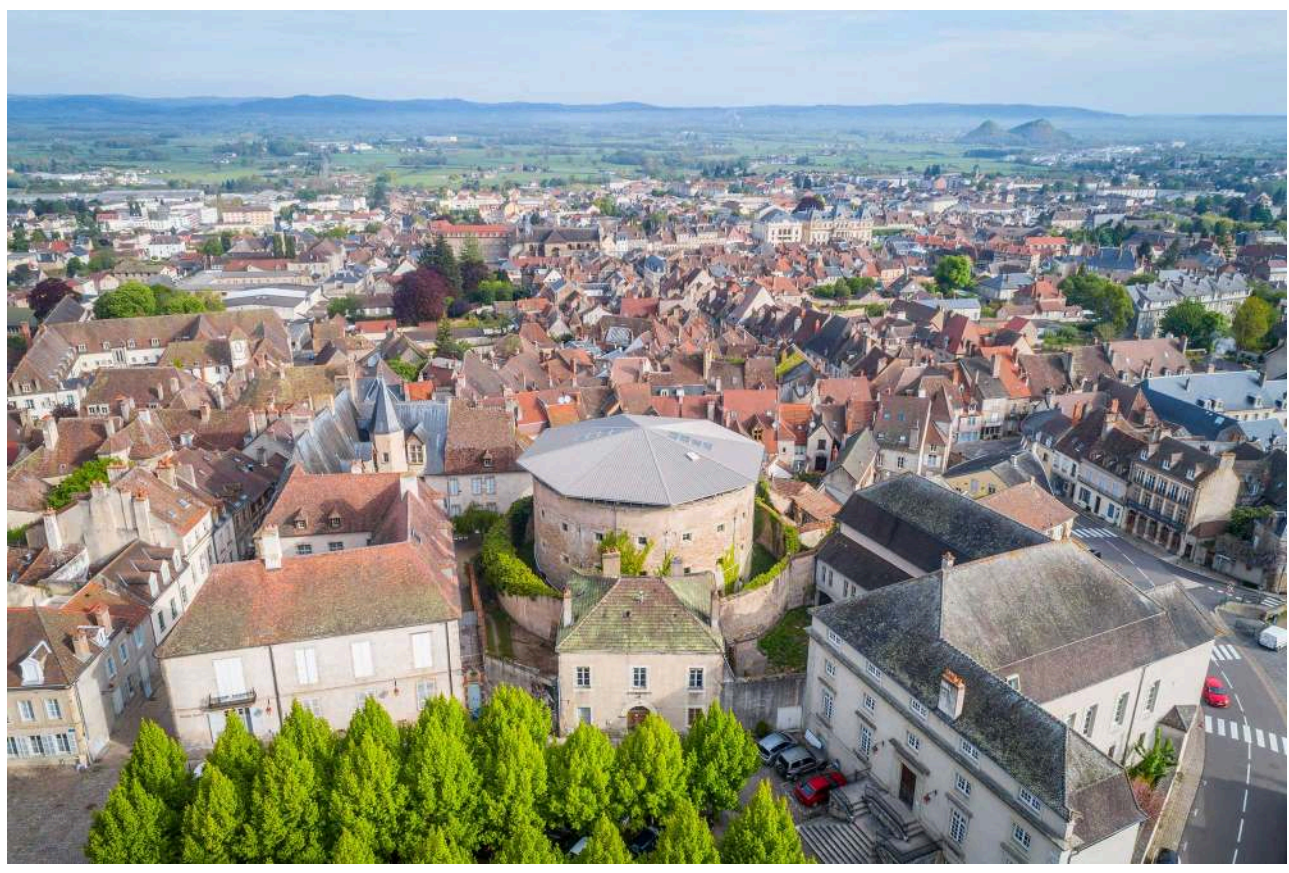

Vue de la place Saint-Louis et des bâtiments concernés par le projet de musée : au centre la prison, à gauche le musée Rolin, à droite le palais de justice.

(c) X. Spertini.

3 Le projet architectural du musée interroge le monument historique et s'apprête à l'adapter à son nouvel usage. C'est l'occasion de se pencher aujourd'hui sur l'architecture de ce lieu, sur les heurs et malheurs de cette petite prison départementale qui a fonctionné durant un siècle selon les dispositions d'un plan initial, souvent critiqué, mais peu modifié jusqu'à la fermeture et le quasi-oubli de ce site après sa fermeture.

\section{La prison circulaire d'Autun : un projet inscrit dans son temps et pourtant unique}

\section{Que remplace la prison circulaire?}

4 Après la Révolution, c'est au conseil général de Saône-et-Loire que fut dévolue la gestion de la prison, alors située dans l'ancien présidial d'Autun. Le présidial devient un palais de justice et la prison s'y trouve réaménagée après quelques travaux. Typique des prisons d'Ancien Régime, elle mêle, dans des cachots insalubres, prévenus et condamnés, hommes, femmes et enfants. De nombreux documents d'archives témoignent durant toute la première moitié du XIXe siècle des critiques qu'elle suscite, des conditions de vie extrêmement difficiles des détenus et des tentatives, jamais abouties, pour y apporter quelques améliorations [fig. 2]. 
Figure 2

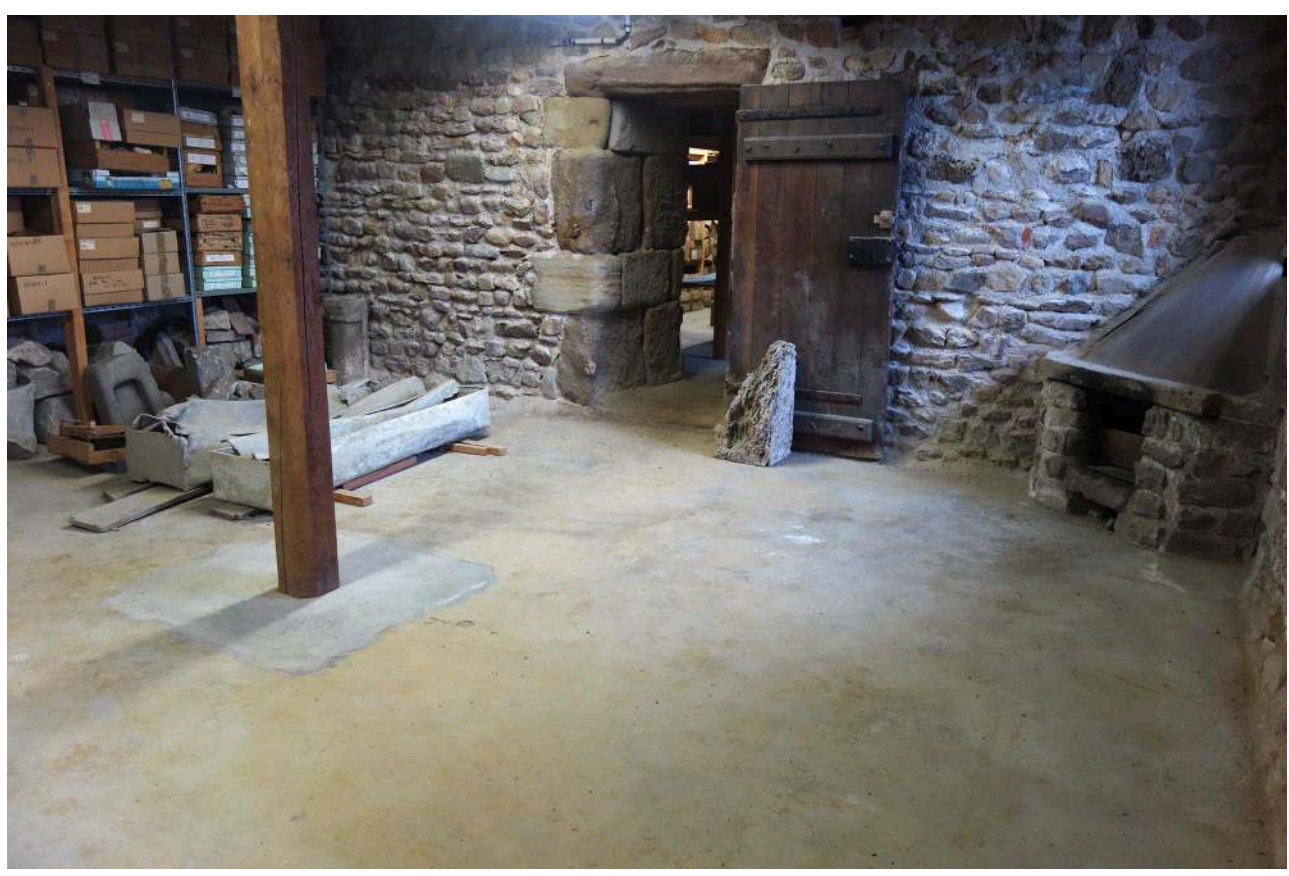

Vue intérieure d'une ancienne salle commune de la prison du palais de justice, actuellement utilisée comme réserve archéologique.

(c) Agathe Legros (Ville d'Autun)

\section{La question de la prison à l'époque du projet}

5 Lorsque l'architecte André Berthier ${ }^{3}$ propose son projet en 1847, c'est en faisant table rase de cet ancien modèle. Il s'appuie alors sur les idées et les débats de son temps, qui dépassent largement le strict champ de l'architecture. Les prisons nouvelles doivent traduire dans leur forme ces grandes théories pour les rendre opérantes sur le quotidien des prisonniers.

6 Attentifs au panoptisme de Jeremy Bentham et aux modèles expérimentés aux ÉtatsUnis, les mouvements philanthropiques français, relayés par la Société royale pour l'amélioration des prisons (créée en 1819), œuvrent sous la Restauration pour la rénovation des prisons ${ }^{4}$. Il s'agit tout autant d'améliorer les conditions d'enfermement des détenus, en limitant leur peine à la stricte privation de liberté, que d'en tirer un meilleur bénéfice social, en opérant sur eux un véritable travail de redressement moral.

7 On cherche à remédier aux défauts des anciennes prisons, qui mélangeaient les détenus dans des salles communes sans pouvoir toujours les distinguer par catégories, en proposant de nouvelles solutions, dont les débats de l'époque se font l'écho. Si certaines questions ne font pas consensus, on peut résumer cependant à quelques préoccupations principales celles qui formeront l'ossature du programme architectural des prisons à partir des années 1830. Dans sa conception nouvelle, le bâtiment doit permettre un emprisonnement individuel par cellule, optimiser la surveillance à la fois dans son efficacité et son économie, renforcer la sécurité, apporter des garanties d'hygiène et de confort minimal, enfin intégrer la discipline et la morale dans le quotidien des prisonniers. 
Sous la monarchie de Juillet, un décret vient préciser et imposer l'idée en fixant le cahier des charges précis de la prison telle qu'elle doit dorénavant être conçue : la circulaire ministérielle de Charles-Tanneguy Duchâtel ${ }^{5}$ est promulguée en $1841^{6}$, assortie d'un atlas de plans proposant différents types de bâtiments adaptés à l'incarcération individuelle. Cette circulaire est destinée à "préparer les moyens d'introduire le régime de l'emprisonnement individuel dans les maisons d'arrêt et de justice. Ce régime, en effet, est le seul que la raison et la justice conseillent d'accorder au prévenu ». Le ministère n'autorise alors plus que la construction de prisons sur ce modèle, largement inspiré des idées de Bentham. Si la tour de surveillance centrale est le pivot du système, la cellule, traduction individuelle d'une prison réservée à chaque prisonnier, y fait l'objet de toutes les attentions.

9 La prison conçue à Autun par l'architecte départemental André Berthier, largement inspirée de cette circulaire, reprend point par point ces préconisations, non seulement dans leurs détails concrets (taille des cellules, hauteur des fenêtres ou du mur du chemin de ronde...), mais aussi dans leur philosophie même, s'attachant à répondre aux problématiques de fonctionnement en prenant certains partis originaux. Mais le projet de Berthier s'inscrit tout d'abord dans un contexte contraint, sur le plan de l'économie, de la localisation, du fonctionnement souhaité, qui oriente largement la réflexion.

\section{Quelle place pour la nouvelle prison d'Autun?}

10 Prenant la mesure des rapports accablants qui se succèdent, le conseil général de Saône-et-Loire sollicite l'architecte André Berthier. La nécessité d'une construction neuve semble alors faire consensus. Sur trois propositions faites par l'architecte - un agrandissement de la prison existante, la construction d'une prison cellulaire ou la construction d'une prison organisée en salles communes-, c'est la proposition cellulaire qui est retenue.

11 Deux emplacements sont alors envisagés, le premier confiné entre le palais de justice et l'ancien hôtel Rolin, l'autre à l'ouest de la cathédrale, à flanc de plateau et dominant la campagne. Le second, plus vaste et isolé, a la préférence de Berthier mais c'est le premier qui est choisi, pour sa proximité immédiate avec le palais de justice, qui permet d'envisager une communication directe entre le tribunal et la prison sans passer par l'espace public. Deux terrains mitoyens sont achetés, pour une somme d'environ $50000 \mathrm{~F}^{7}$, dégageant un espace qui s'étend de la place Saint-Louis aux vestiges d'un rempart médiéval formant terrasse.

Avec cet emplacement, la principale préoccupation de l'architecte est d'isoler les prisonniers de la ville afin d'éliminer toute perception visuelle ou sonore, dans un sens comme dans l'autre. Il imagine alors un bâtiment administratif carré, avec une façade sur la place Saint-Louis qui vient faire écran, empêchant que l'on ne voie ou entende la prison depuis la rue. Deux murs relient de part et d'autre la façade de ce pavillon administratif aux bâtiments adjacents, rendant la prison très peu perceptible depuis l'espace public [fig. 3]. 


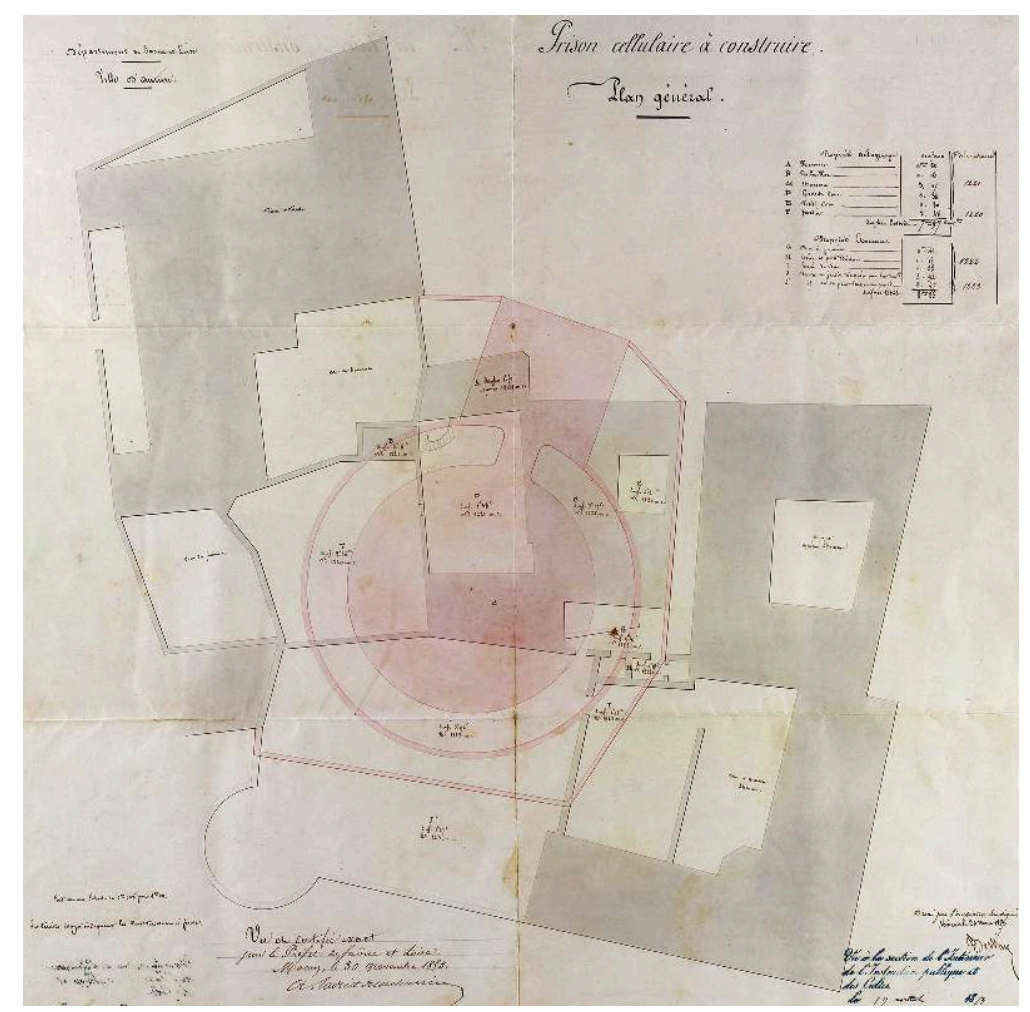

Intégration du plan de la prison au site existant, plan conservé aux archives départementales de Saône-et-Loire (série 4N28-02, Berthier, 1853).

Reproduction AD71.

\section{Application d'un modèle et création originale}

André Berthier s'est, fort logiquement et de manière évidente, inspiré de la circulaire de 1841 pour concevoir sa prison. Le caractère marquant de son projet est sa grande simplicité géométrique et sa pureté architecturale. Il est non seulement fidèle à l'esprit de la circulaire Duchâtel, proscrivant les éléments ostentatoires (grilles, portes impressionnantes...), mais il va même plus loin en proposant un bâtiment d'une remarquable compacité, sans aucune rupture de la ligne générale.

Le pavillon rectangulaire, réservé aux fonctions administratives, est traversé d'un couloir axial qui dessert différentes salles de service. À l'étage se trouve l'appartement du gardien-chef. Un chemin de ronde, large de 3,80 m et ceint d'un mur haut de $5 \mathrm{~m}$, sépare le pavillon des gardiens de la tour des prisonniers. Il isole complètement cette tour de l'extérieur et permet la surveillance tout autour du bâtiment. Enfin, la tour, haute de $13 \mathrm{~m}$ et large de $23 \mathrm{~m}$, rassemble dans un plan circulaire cinquante emplacements de cellules (dont trois utilisées à d'autres fonctions), réparties sur trois niveaux ${ }^{8}$.

On retrouve dans ce bâtiment tous les détails d'aménagements que la circulaire de 1841 avait soigneusement mentionnés. La dimension des cellules, la disposition des fenêtres et leur ouverture à distance par les gardiens, la présence de sanitaires couplés de part et d'autre des cloisons séparant les cellules, les doubles-portes (une pleine et une grillagée) répondent de façon minutieuse à ses préconisations. 
Pourtant, le projet d'Autun se distingue en plusieurs points des prisons proposées en modèle. Avec à peine cinquante cellules, c'est une prison relativement modeste. Sa petite taille a pu contribuer au choix du plan circulaire, souvent cité mais peu appliqué en raison de sa complexité de mise en œuvre et de son coût. Ici, le petit nombre de cellules permet la couverture de l'édifice par une coupole de taille raisonnable, audessus d'une cour ronde large d'une douzaine de mètres. Chaque cellule, voûtée, s'appuie sur ses voisines de part et d'autre et sert de contrebutement à la coupole, le tout formant un ensemble d'une grande stabilitég [fig. 4].

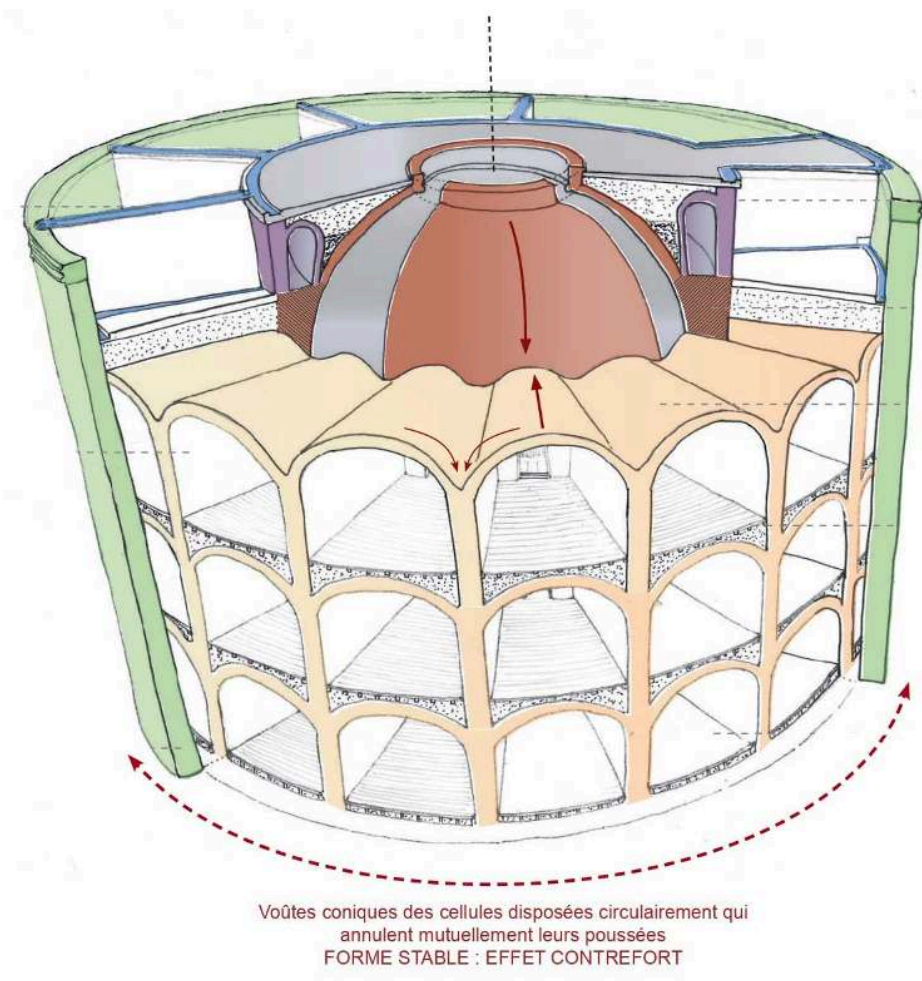

Dessin analytique du principe architectural de la prison d'Autun, réalisé dans le cadre d'un projet d'étude à l'École de Chaillot, 2016.

(C) Lucille Cau.

17 Autre différence notable, l'absence de tour de surveillance: ici l'espace central, abondamment baigné de lumière grâce à l'oculus placé au sommet de la coupole, est laissé libre de toute construction et n'est destiné qu'aux services religieux. En effet, c'est un autel qui doit prendre place au centre de la tour de la prison, autre réponse donnée à l'injonction de permettre à tous les détenus de suivre la messe depuis leur cellule.

Les habituels préaux et cours de promenade semblent à première vue absents, mais c'est sans doute l'une des innovations d'A. Berthier que d'avoir imaginé les positionner au sommet du bâtiment. Au-dessus du dernier niveau de cellules sont placées huit cours de promenade à ciel ouvert, ceintes de murs de $2 \mathrm{~m}$ de haut interdisant tout regard sur la ville et desservies par une galerie courant sur le flanc de la grande voûte. Les gardiens ont accès au toit-terrasse surplombant ces cours, toit depuis lequel ils peuvent effectuer leur surveillance. 
peut enfin noter, malgré sa sobriété affichée, la réalisation soignée du bâtiment, dont le coût s'élève à $115974 \mathrm{~F}$. Certes, la maçonnerie est majoritairement faite de moellons, mais le pavement de pierres soigneusement appareillées, les cellules dotées de plancher, le système sophistiqué de chauffage et d'évacuation des eaux usées, la régularité et les proportions très équilibrées du bâtiment trahissent l'ambition de l'architecte. Inspiré de l'architecture classique, il concentre son effort sur l'intégration la plus complète du programme à un volume "parfait ", dont la simplicité et la clarté rejoignent le rêve de J. Bentham d'une prison signifiant sa fonction par sa forme même : «Cette prison bâtie sur le principe panoptique est transparente, ouverte à tout le monde. Il suffit, en quelque manière, d'un seul coup d'œil pour la voir tout entière ${ }^{10} . »[$ fig. 5$]$.

Figure 5

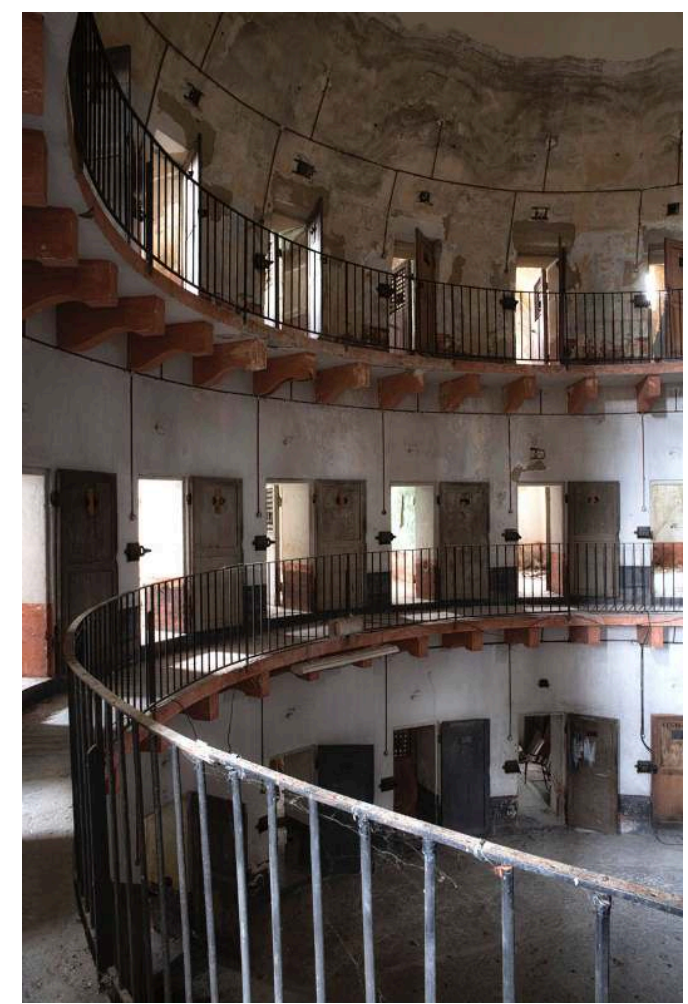

Vue intérieure de la prison depuis la coursive du premier étage, 2019.

(c) Didier Verriest, voir didierverriest.com [lien valide en janvier 2002].

\section{À l'épreuve de la réalité}

\section{Une prison déjà caduque?}

La prison d'Autun doit à la durée de son chantier d'être, quasiment dès son ouverture, inadaptée aux mesures préconisées par l'État. Si le plan réalisé en 1847 est bien validé, sa mise en œuvre est interrompue en 1848 puis réétudiée en 1852, durant les premières années du Second Empire, marquées par une forte augmentation du nombre de prisonniers. Le chantier prend encore quelques années et la prison accueille ses 
premiers détenus en 1857, dix ans après les premiers dessins de l'architecte. Or ces dix années ont connu de profonds changements politiques et sociaux.

Le projet de 1847 était en phase avec les idées de la monarchie de Juillet et se voulait résolument moderniste mais le tournant politique du Second Empire conduisit à aborder le problème d'une manière beaucoup plus pragmatique et économique. Constatant la portée limitée de la circulaire Duchâtel, une nouvelle circulaire du ministre de l'Intérieur Persigny ${ }^{11}$, éditée en 1853, abandonne et même déconseille le modèle de l'emprisonnement en cellules individuelles, jugé trop coûteux et irréaliste. Cette circulaire revient à une séparation par quartiers des catégories de détenus, qui ne nécessite que des aménagements mineurs par rapport aux modèles des prisons d'Ancien Régime. Elle réclame également l'établissement d'ateliers, espaces complètement absents du projet d'A. Berthier, pour ménager aux détenus des temps de travail. La prison d'Autun se trouve ainsi, dès son ouverture, décalée par rapport aux idées de son temps. L'aboutissement de son plan, réponse parfaite au programme de l'enfermement individuel, ne permet guère une adaptation a posteriori à un autre fonctionnement. Seules quelques cellules seront par la suite réaffectées, d'une part en infirmerie, d'autre part en deux zones d'ateliers ménagées sur cinq cellules décloisonnées au rez-de-chaussée.

\section{Premiers désagréments}

Au-delà de cette adéquation ou non aux directives en vigueur, d'autres problèmes adviennent à peine la prison ouverte, dont nous informe la correspondance administrative conservée.

Une lettre du gardien-chef, du 15 septembre $1857^{12}$, fait part de ses critiques sur ce nouveau bâtiment et les défauts de cette prison neuve, récapitulés en dix points. Ils ont trait pour la plupart à des problèmes de sécurité, notamment la faiblesse des serrures, mais aussi la présence de tuyaux pouvant faciliter l'escalade des murs. Les aménagements « de confort " sont jugés insuffisants: les cellules ont un lit pour tout mobilier et la note réclame instamment l'ajout d'une assise et d'un rayonnage qui permette aux détenus de poser affaires et nourriture ailleurs qu'au sol. Les vasistas, conçus pour être manipulés depuis l'extérieur de la cellule, sont impossibles à rouvrir une fois fermés faute d'un contrepoids suffisant. Plus problématique peut-être à long terme, le bâtiment lui-même accuse quelques faiblesses et le gardien-chef pointe des évacuations insuffisantes et rapidement engorgées, mais aussi des fuites par la toiture, notamment le lanterneau. Il précise qu'il pleut sur l'autel, ce qui indique au passage la présence effective de celui-ci au centre de la rotonde dès l'ouverture de la prison.

Les imperfections d'un bâtiment qui vient d'ouvrir se mêlent ici à des désordres plus graves qui ne cesseront d'être rappelés jusqu'à la fermeture de la prison, à commencer par des problèmes d'étanchéité et d'évacuation. Petits ou grands, aucun de ces problèmes ne semble donner lieu à une intervention et la réponse d'A. Berthier, si elle ne se fait pas attendre (courrier du 28 septembre $^{13}$ ) en fait peu de cas. Il admet seulement la nécessité de compléter le mobilier des cellules et demande en 1858 un crédit de 1000 francs pour pourvoir les 48 cellules de bancs, tables et planches à pain, sans que cela ne lui soit a priori accordé. 


\section{L'affaire de l'autel}

L'une des grandes originalités de la prison d'Autun par rapport au modèle de Bentham tient à l'absence de tour de surveillance centrale, remplacée ici par un autel. Il apparaît clairement sur les plans d'A. Berthier que l'espace central est à l'origine laissé vide de toute construction, ce que confirme d'ailleurs son rapport accompagnant le projet ${ }^{14}$. En 1858 , les travaux tout juste achevés, l'évêque s'émeut de l'emplacement de l'autel, au rez-de-chaussée, et demande sa surélévation. Cette modification du projet, chiffrée à 3000 francs, est soumise au conseil général par le conseil d'arrondissement d'Autun ${ }^{15}$ et figure au budget de $1860^{16}$.

Aujourd'hui, huit traces d'arrachement au centre de la rotonde rappellent la présence d'une structure octogonale, reposant sur huit pieds de faible empiètement [fig. 7], ce qui laisse supposer une construction de métal. La description qui en est faite dans le budget de 1860 précise qu'il s'agit d'une plateforme à hauteur du premier étage, reliée à celui-ci par une passerelle. En dessous est ménagé un espace vitré destiné à la surveillance des prisonniers.

Aucun dessin, aucune photo ancienne de cette tour centrale n'est connu à ce jour, mais elle figure sur les plans du bâtiment dressés en 1876. D'après un témoignage oral ${ }^{17}$, elle aurait été démantelée peu après le rachat du site par un propriétaire privé, dans les années 1950 ou 1960 [fig.6].

Figure 6

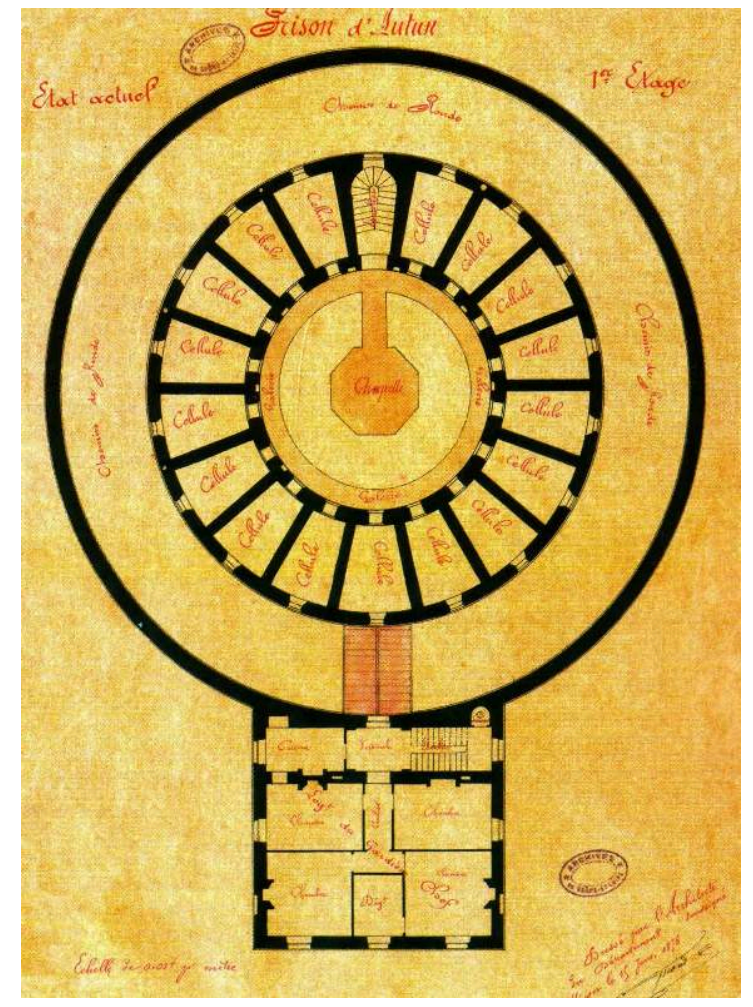

Plan de la prison sur lequel on observe le plan octogonal de la plateforme centrale, relié par une passerelle à la galerie du premier étage côté nord, conservé aux archives départementales de Saôneet-Loire (série 4N28, Jean-Etienne Giroud, 1877). Cette plateforme n'est pas figurée sur la coupe du bâtiment réalisée en même temps.

Reproduction AD71. 


\section{Les préaux, principal défaut de la prison}

ajustements d'un bâtiment neuf durant ses premières années de fonctionnement, il reste un sujet qui constitue la principale faiblesse du site, du point de vue de la sécurité mais aussi pour la bonne conservation du bâtiment lui-même : les cours des préaux. Ces préaux, situés à l'étage supérieur de la prison, accolés à la coupole et à ciel ouvert, sont sans doute la principale originalité du projet d'A. Berthier mais aussi son grand défaut [Fig. 7].

Figure 7

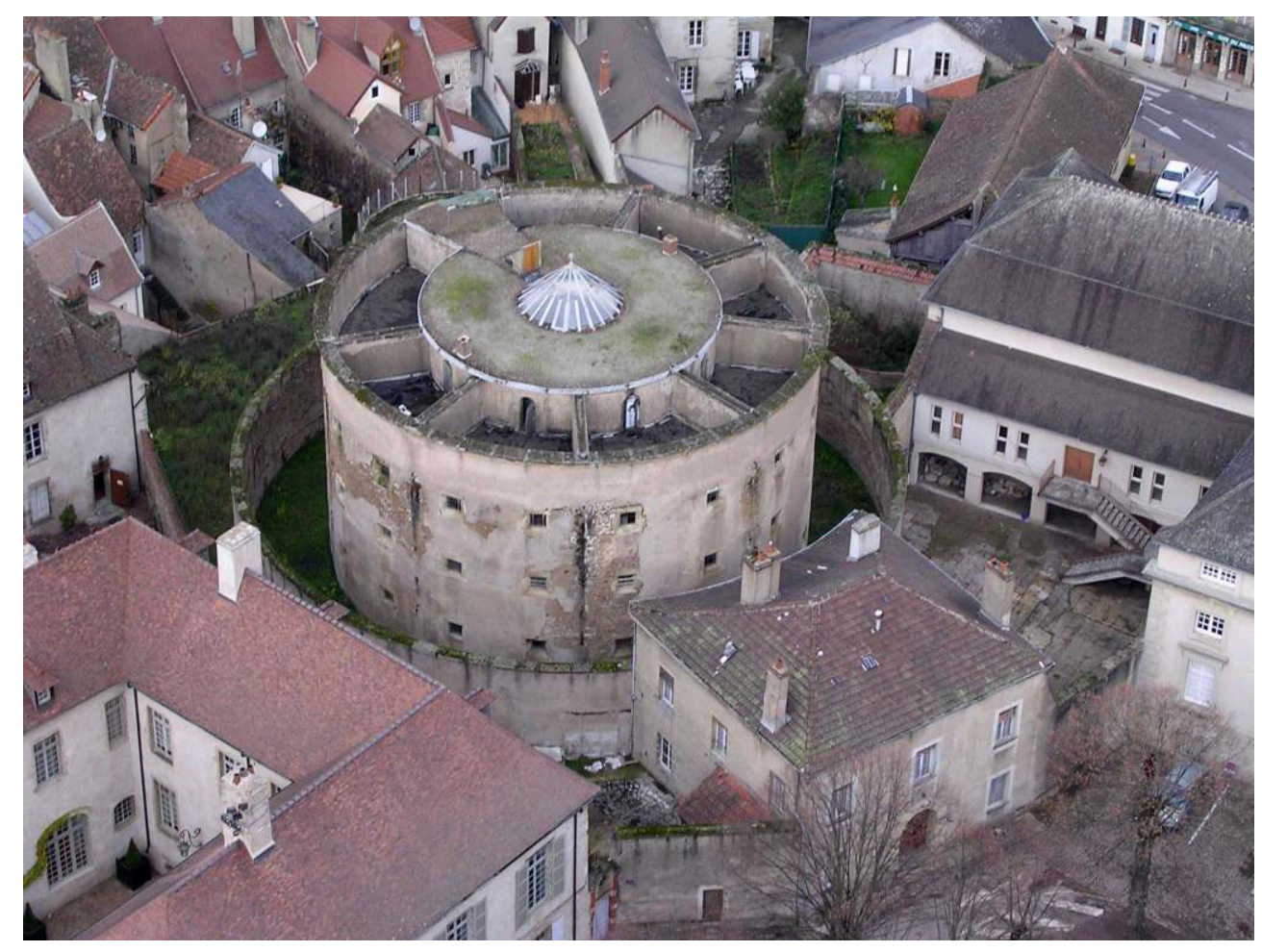

Vue de la prison avant pose de la toiture provisoire, où l'on observe les huit cours de promenade dominées par un petit toit terrasse dont le centre est occupé par le lanterneau.

(c) Danièle Thévenin (Ville d'Autun).

Dès 1858 , des alertes sont données sur le caractère peu sécurisé de ces espaces, clos de murs faciles à escalader, desservis par un couloir trop exigu pour permettre un encadrement correct des détenus. Ils sont par ailleurs d'usage malcommode, sans protection contre le soleil et les intempéries, théoriquement surveillés par des gardiens positionnés sur le toit-terrasse en surplomb, eux-mêmes encore plus exposés aux aléas du temps. Enfin et surtout, malgré les caniveaux et descentes d'eaux pluviales encastrées dans les maçonneries de façade, ces préaux sont rapidement à l'origine de nombreuses infiltrations. Les sols sont recouverts d'un bitume de schiste, qui se dégrade sous l'action de l'eau et du gel, laissant l'eau s'infiltrer dans les murs de la tour centrale. Les problèmes d'évacuation des eaux de pluie recueillies dans les préaux contribuent à la dégradation du bâtiment jusqu'au début des années 2000, causant la destruction de la quasi-totalité des enduits extérieurs et entrainnant des désordres dans 
les maçonneries. Ce n'est qu'avec la mise en place d'une toiture provisoire en 2013, couvrant l'ensemble de la tour centrale, préaux compris, que le bâtiment a pu être progressivement assaini.

A. Berthier a tenté d'améliorer cette question précise des préaux, prenant en compte leur faiblesse en termes de sécurité, d'usage, d'étanchéité, mais aussi pour répondre aux dernières directives de la circulaire Persigny. Dès 1858, il propose une modification de son projet pour un coût de $11000 \mathrm{~F}^{18}$. Les aménagements projetés, dont les plans ne semblent malheureusement pas avoir été conservés, consistent à couvrir quatre préaux, les mettre en communication et les chauffer pour qu'ils puissent servir d'ateliers. Les quatre autres gardent leur vocation de cour de promenade mais sont recouverts de treillages, pour protéger les détenus du soleil et empêcher toute tentative d'évasion. Ce projet est refusé. Il est décidé à la place de créer des ateliers dans l'espace de l'ancienne prison ${ }^{19}$.

\section{Tentatives de restauration}

31 Passé les vagues de critiques et amendements accompagnant son ouverture, la prison a fonctionné sans aménagements autres que la surélévation de l'autel et la dévolution de certaines cellules du rez-de-chaussée aux fonctions d'infirmerie et d'ateliers. Des ateliers sont aménagés dans la cour du tribunal jouxtant le bâtiment. Tout au long du $\mathrm{XIX}{ }^{\mathrm{e}}$ siècle, les archives témoignent de la persistance des problèmes d'évacuation des eaux usées, d'infiltrations par les préaux de promenade, mais aussi de stabilité des sols mal remblayés, causant des lézardes aux murs.

La loi du 5 juin $1875^{20}$, complétée par la circulaire du 27 juillet $1877^{21}$, suscite apparemment une dernière tentative de modification d'envergure de la prison panoptique. Ces textes instituent définitivement l'emprisonnement individuel pour les courtes peines mais confirment aussi les ateliers communs et demandent des cours de promenade plus vastes. L'économie du bâtiment est prise en compte, avec des préconisations, par exemple, en faveur d'un chauffage à eau chaude plutôt qu'à air chaud.

À Autun, l'architecte départemental Jean-Étienne Giroud rend le 10 janvier $1879^{22}$ un constat à charge du bâtiment, tant du point de vue du fonctionnement que de la qualité architecturale. On y lit que les préaux, trop malcommodes, n'ont jamais été utilisés comme tels, que l'eau s'y infiltre et cause la ruine du bâtiment, que la poussée de la voûte centrale est excessive et entraîne des désordres dans les maçonneries. Il convient à la fois de remédier à ces problèmes structurels, de s'aligner sur les consignes de la loi de 1875 et de mieux organiser l'accueil des détenues.

\section{Projet de 1876}

34 Un premier projet, en 1876, [fig. 8 et fig. 9] avait envisagé une extension importante du bâtiment vers le nord, c'est-à-dire au fond de la parcelle du site, avec l'adjonction d'un bâtiment spécifique destiné aux femmes, comprenant quatre cellules au rez-dechaussée et quatre cellules à l'étage. La tour circulaire, pensée pour être tout orientée vers son centre et ceinte de trois clôtures successives, se trouve ici largement ouverte selon un axe longitudinal. Les cours de promenade au niveau supérieur sont couvertes d'une toiture et deviennent de simples espaces sous combles. De nouveaux préaux sont 
aménagés de plain-pied, ceux des femmes empiètent sur le chemin de ronde. De cette version proviennent également des détails d'aménagement de confort (eau, gaz) et des propositions de mobilier [fig. 10]. Le fonctionnement panoptique du bâtiment circulaire passe ici largement au second plan, de même que son principe de surveillance par le chemin de ronde.

\section{Figure 8}

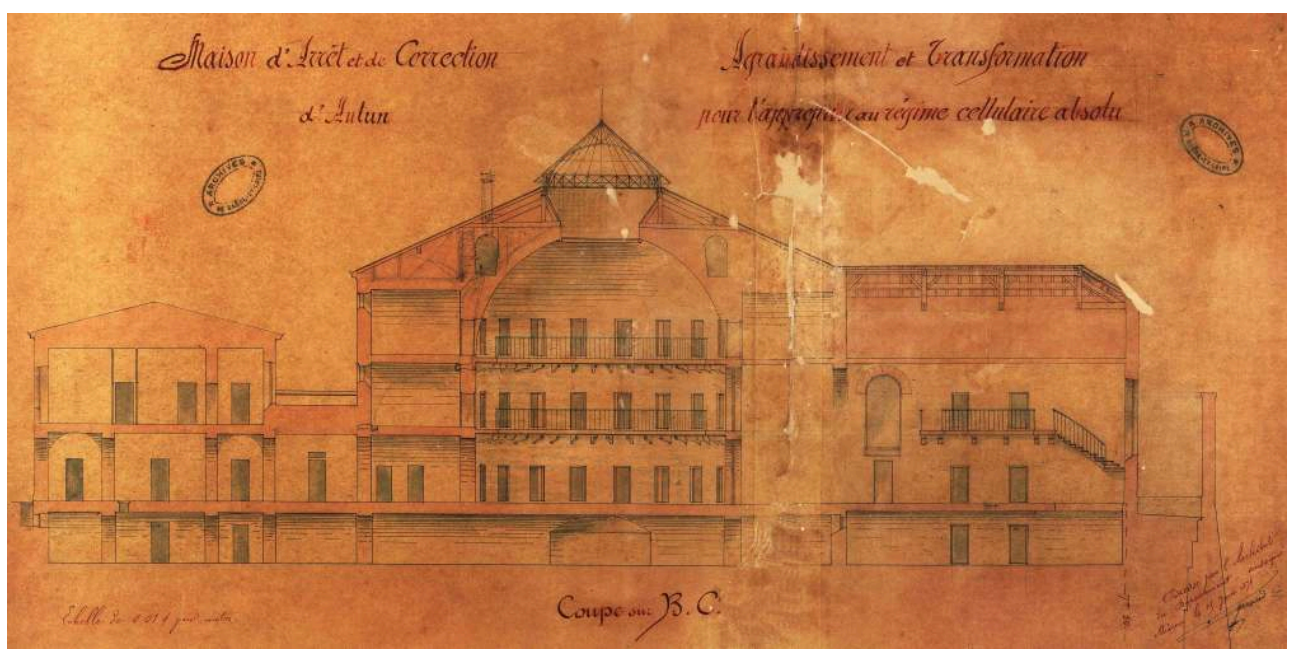

Coupe du projet de 1876, J.-E. Giroud, conservée aux archives départementales de Saône-et-Loire (série 4N28).

Reproduction AD71. 


\section{Figure 9}

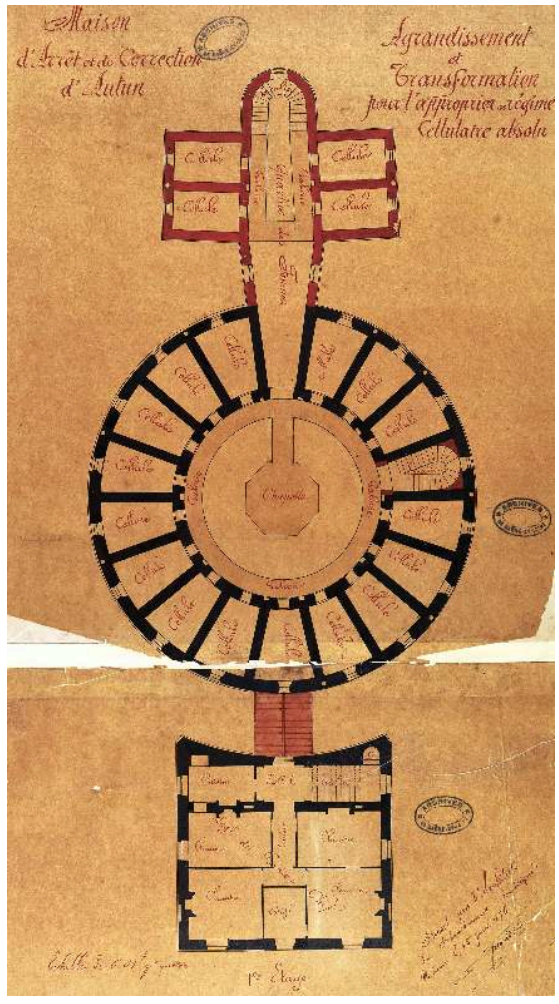

Plan du projet de 1876 au niveau du premier étage, J.-E. Giroud, conservé aux archives départementales de Saône-et-Loire (série 4N28).

Reproduction AD71.

\section{Figure 10}

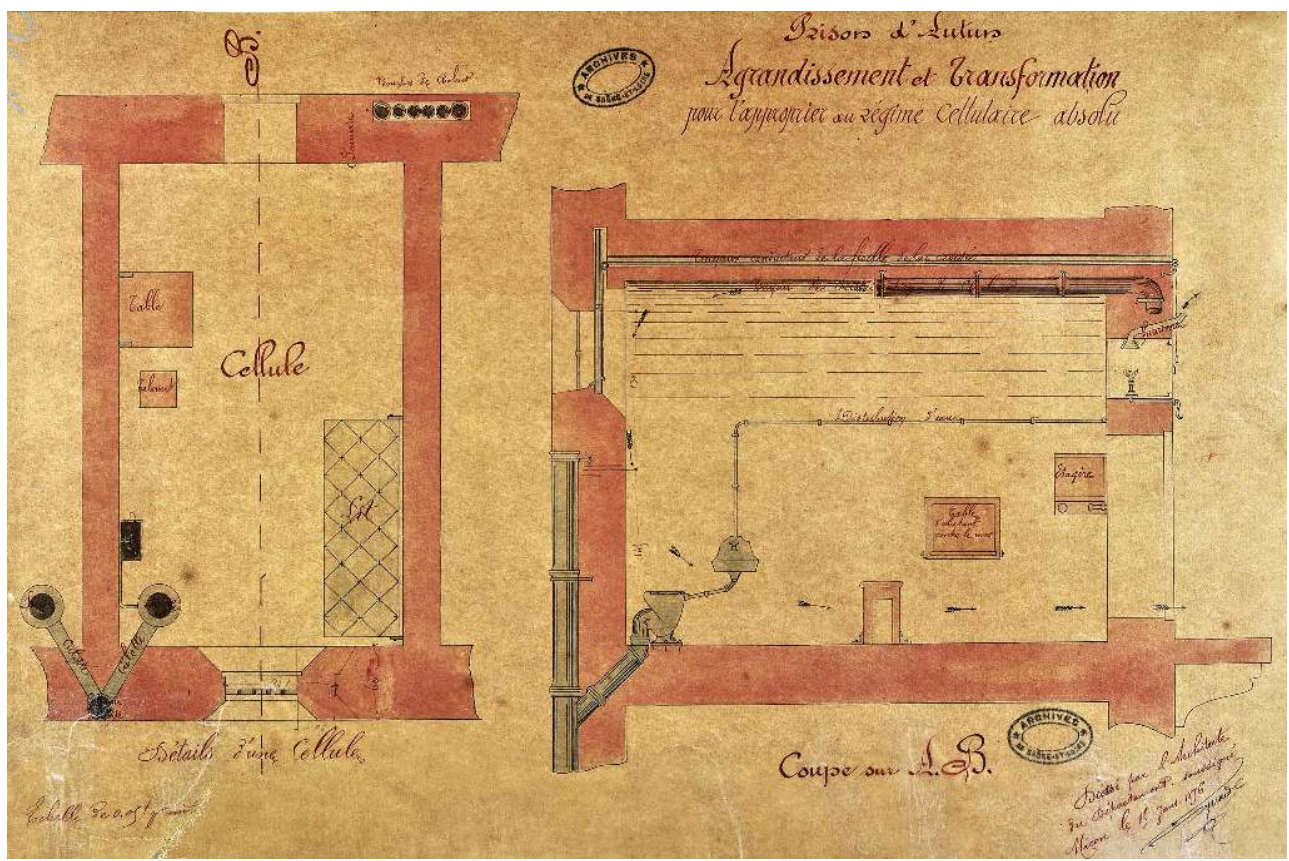

Coupe et plan d'une cellule de la prison circulaire avec les nouveaux aménagements, J.-E. Giroud, conservés aux archives départementales de Saône-et-Loire (série 4N28).

Reproduction AD71. 


\section{Projet de 1879}

supprimé au profit de deux plus petits, installés de part et d'autre de la tour. Le rapport de l'architecte nous apprend qu'ils fonctionnent à l'eau chaude, comme le préconise la nouvelle législation. Chacun dessert un quartier de détention, celui des femmes du côté du pavillon rectangulaire, celui des hommes à l'opposé. Les plans du rez-de-chaussée et des étages détaillent l'installation du quartier des femmes. Au rez-de-chaussée [fig. 11], un escalier spécifique dessert les cellules à l'étage et trois préaux sont prévus entre le pavillon des gardiens et le palais de justice.

Figure 11

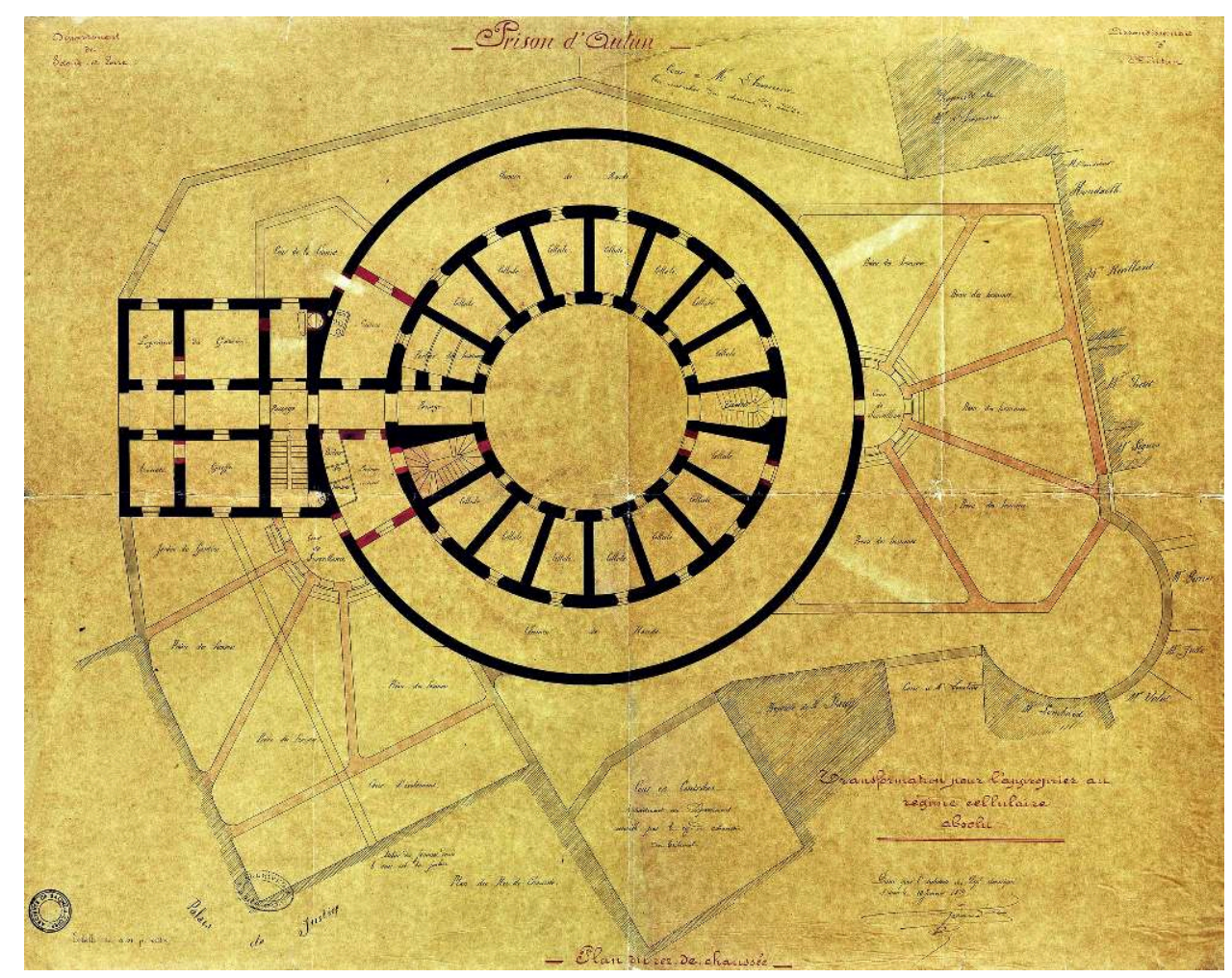

Projet de 1879, plan du rez-de-chaussée, J.-E. Giroud, conservé aux archives départementales de Saône-et-Loire (série 4N28).

Reproduction AD71.

Les ateliers sont alors apparemment conservés dans la cour du tribunal, là où ils avaient été précédemment aménagés. Entre l'escalier et les préaux, à l'emplacement du chemin de ronde, un petit parloir est dessiné. Aux étages, les cellules sont occultées aux regards depuis le quartier des hommes par des persiennes en menuiserie de chêne, qui bordent le parapet [fig. 12]. Le principe panoptique du bâtiment, de même que l'organisation initiale de sa surveillance, sont ici superbement ignorés. Cependant la 
chapelle reste centrale et cette galerie pourvue de persiennes doit permettre aux femmes de suivre les offices sans voir ou être vues du quartier des hommes.

Figure 12

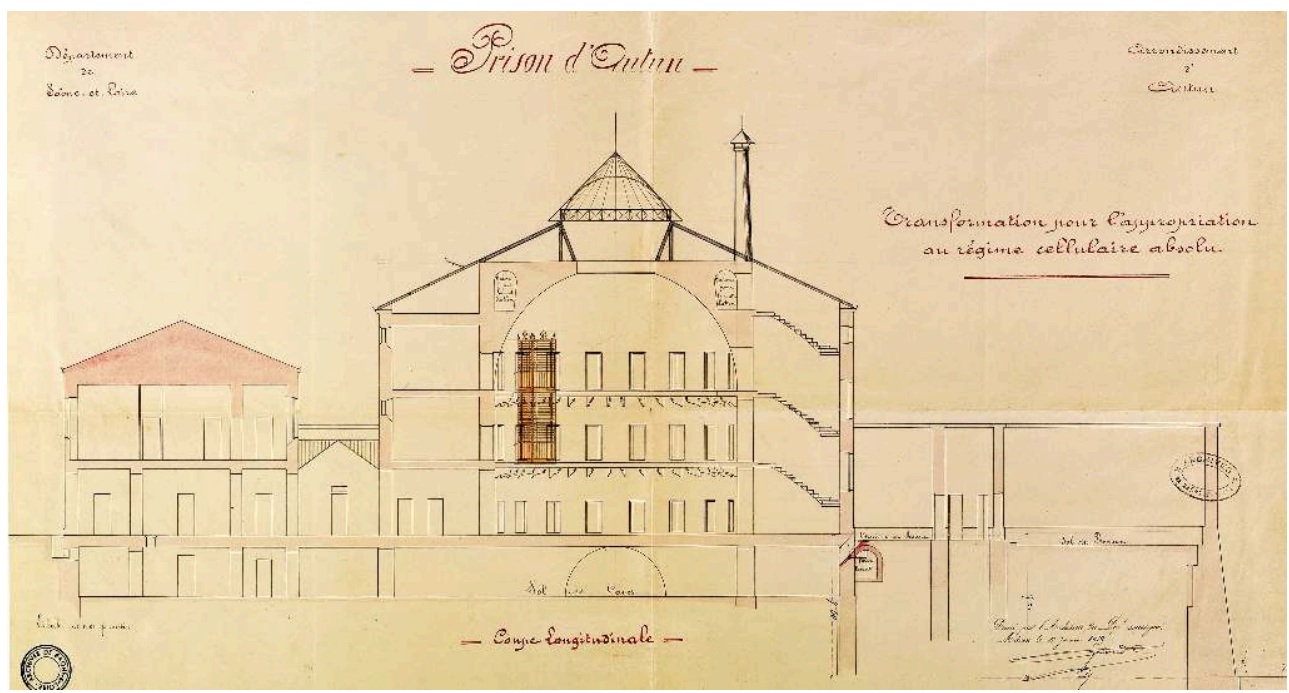

Projet de 1879, coupe longitudinale, J.-E. Giroud, conservée aux archives départementales de Saôneet-Loire (série 4N28)

Reproduction AD71.

Enfin, les préaux deviennent des espaces sous combles [fig. 13], leurs murs de refends sont doublés pour conforter la voûte et l'on y installe deux réservoirs, destinés à fournir l'eau à l'ensemble du bâtiment, y compris dans les cellules. 


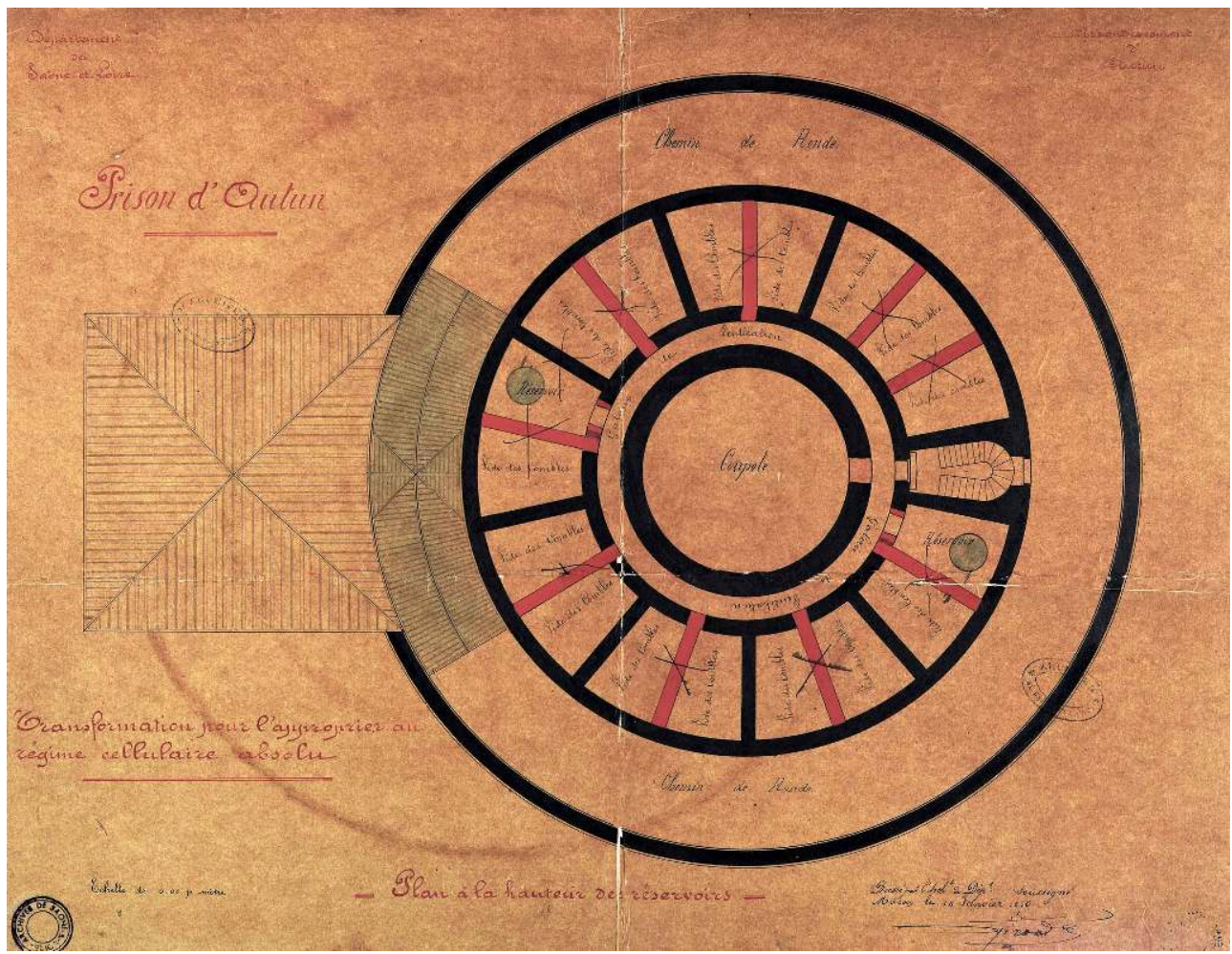

Projet de 1879, plan du troisième étage, niveau des préaux, J.-E. Giroud, conservée aux archives départementales de Saône-et-Loire (série 4N28).

Reproduction AD71.

Ces deux projets successifs montrent à quel point la recherche de solutions pragmatiques s'éloigne alors des principes constitutifs du bâtiment, que ce soit sur le plan architectural ou fonctionnel. Dans les deux cas, la couverture des préaux est prévue, répondant à ce qui semble être le principal problème du bâtiment. L'intégration des femmes met à mal le principe panoptique : dans la tour circulaire, les détenus ont une vision quasi globale sur le bâtiment depuis leur cellule si la porte pleine est ouverte. Cette disposition semble incompatible avec la présence d'un quartier de détenues, que chacun des deux projets veille à rendre autant que possible indépendant $\mathrm{du}$ quartier des hommes. La suppression des cours de promenade du $3^{e}$ niveau nécessite de créer de nouveaux espaces "en pétales" logés dans les interstices encore disponibles du terrain de la prison. Le chemin de ronde est lui aussi largement sacrifié, occupé selon les cas par des espaces de service (cuisine, parloir) ou des cours de promenade.

\section{Fin de la prison, naissance d'un lieu de patrimoine}

Le projet de 1879 n'est pas réalisé et seuls les travaux d'entretien les plus courants sont engagés. La prison fonctionnera encore quelques décennies dans un bâtiment finalement inchangé depuis sa conception de 1847. Fermée officiellement à partir de 1934, réutilisée sous l'Occupation et à la Libération, elle ne connut pas d'autre usage susceptible d'en modifier profondément son architecture. Seuls les dommages du temps, avec l'aggravation des infiltrations et des désordres conséquents sur le 
bâtiment, et les dégradations humaines (démontage des éléments métalliques, graffitis...) vont peu à peu l'altérer.

Une lente prise de conscience de l'intérêt du lieu est cependant à l'œuvre et la prison est inscrite à l'Inventaire supplémentaire des monuments historiques en 1975. Après quelques décennies en mains privées, la prison est acquise par la Ville d'Autun en 2003. D'emblée, le projet est de l'intégrer à l'extension future du musée Rolin. Un usage culturel du site est organisé durant quelques années, avec l'accueil d'expositions et d'un espace réservé à l'école municipale de dessin, jusqu'à ce que la dégradation du bâtiment interdise d'y recevoir du public. En 2013 est installée la toiture provisoire couvrant le lanterneau et les préaux, mettant fin aux infiltrations dont souffrait le bâtiment depuis un siècle et demi.

La programmation d'une extension du musée Rolin, engagée à partir de 2015, englobe à la fois l'hôtel Rolin, siège du musée actuel, la prison panoptique et le palais de justice ${ }^{24}$. Ce programme prend en compte la dimension patrimoniale de la prison et préserve largement ses dispositions architecturales intérieure et extérieure. Une séquence muséographique dédiée à l'interprétation du bâtiment est incluse dans le projet muséographique. L'évolution de son usage est cependant dessinée, avec entre autres la nécessité d'améliorer l'accessibilité des différents niveaux, la couverture du chemin de ronde pour gagner en superficie d'exposition et surtout, le souhait de créer une extension de la prison en surélévation. Ce niveau panoramique, couvrant les cours des préaux et dominant le paysage urbain, transforme la prison en un espace de découverte et d'interprétation de la ville par son paysage et son histoire.

En 2020, l'Atelier Novembre est désigné lauréat du concours, posant à son tour de nouvelles questions sur l'évolution du bâtiment. Comment créer une circulation simple dans un lieu destiné à l'enfermement? Comment faire d'un lieu discret, sinon caché dans le paysage urbain, le cœur d'un musée ouvert sur la ville? Comment préserver cette prison unique, la donner à voir, tout en l'adaptant à un usage complètement différent? [fig. 14]. 
Figure 14

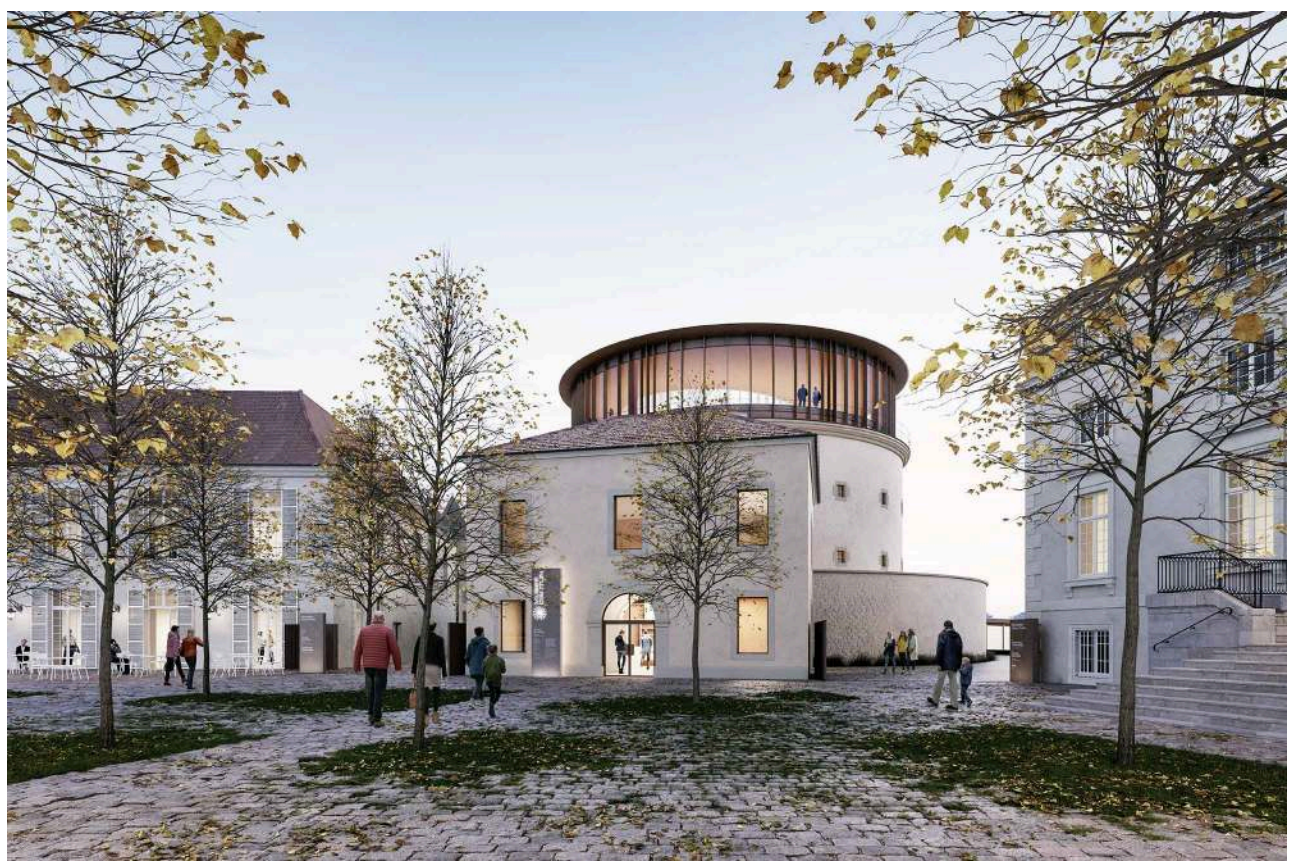

Projet de l'Atelier Novembre, perspective donnant à voir l'entrée du musée depuis la place Saint-Louis, 2019.

(c) Alexandre Besson.

Le projet ose plusieurs partis ambitieux. Le bâtiment lui-même est rendu visible et intégré à la ville par la suppression des murs qui clôturent le site. La parcelle de la prison devient un espace ouvert et public. L'aménagement intérieur du pavillon des gardiens est modifié, avec notamment le décloisonnement des espaces, pour en faire le point d'entrée principal du site et son accueil. Le chemin de ronde est couvert et initie le parcours de visite, menant à un vaste niveau inférieur qui relie la prison aux autres bâtiments. La tour centrale est largement préservée, bien que certaines cellules doivent laisser place aux aménagements de confort et de circulation du public. Enfin, l'étage supplémentaire, surélévation en extension coiffée d'une toiture métallique, vient se poser sur le bâtiment initial, tout en préservant le principe de l'oculus central.

Ce projet, retenu par la Ville d'Autun, en accord avec ses partenaires, répond au programme d'un musée en ayant le double objectif de présenter des collections exceptionnelles et d'apporter une interprétation et une compréhension de l'histoire de la Ville. La prison en devient le symbole et le point d'entrée, tout en assumant son identité de bâtiment carcéral. C'est maintenant dans un travail fin et sensible de scénographie qu'il faut s'engager, pour offrir aux visiteurs de retrouver la prison panoptique préservée et mise en valeur au sein du musée. 


\section{NOTES}

1. Je voudrais adresser mes remerciements les plus vifs à Isabelle Vernus, directrice des archives départementales de Saône-et-Loire, pour les informations qu'elle m'a communiquées, ses conseils attentifs et précis et pour m'autoriser à publier ici les plans expressément numérisés par son équipe ; Jean-Baptiste Rezvoy, ingénieur du patrimoine, pour la documentation qu'il m'a fournie et son regard complémentaire sur ce dossier; André Strasberg, conservateur en chef, pour les informations partagées sur la prison du palais de justice et l'histoire de la prison circulaire après sa fermeture.

2. Arrêté de classement du 9 juin 2017 portant sur la totalité de la prison et de son assise. Consultable sur la base Mérimée, notice PA00113095, voir https://www.pop.culture.gouv.fr/ gallery/60d99d55a82c630e0f5ac512 [lien valide en septembre 2021].

3. André Berthier, alors jeune architecte à Charolles, est sollicité à cette époque par le conseil général de Saône-et-Loire pour concevoir la nouvelle prison d'Autun. Il deviendra architecte départemental en 1850 et le restera jusqu'en 1862.

4. L'essentiel des informations résumées dans ce court paragraphe historique est tiré de: CARLIER Christian, "La construction des prisons en France au xIX ${ }^{\mathrm{e}}$ siècle: de longues hésitations ", Musée Criminocorpus, article publié à l'occasion de l'exposition en ligne " Histoire des prisons de Lille» en 2009, Paris, Centre pour les humanités numériques et l'histoire de la justice, 2009, [en ligne] https://criminocorpus.org/fr/ref/25/17367/ [lien valide en septembre 2021].

5. Ministre de l'Intérieur du gouvernement de François Guizot de 1840 à 1848.

6. Voir https://criminocorpus.org/en/landmarks/legislation/textes-juridiques-lois-decre/ textes-relatifs-aux-p/de-la-monarchie-de-juillet-a-1/9-aout-1841-circulaire-pour-1/ [lien valide en septembre 2021].

7. Une ancienne maison canoniale à trois ailes sur cour, bordant la place Saint-Louis, appartenant à un M. Lagrange et un espace de jardin associé à l'hôtel Rolin, propriété de M. Lhomme de Mercey.

8. Le bâtiment et sa disposition intérieure sont précisément décrits dans : FOUCART Bruno \& NOEL-BOUTON Véronique, «Une Prison cellulaire de plan circulaire au XIX siècle: la prison d'Autun », L'Information d'histoire de l'art, Paris, $\mathrm{n}^{\circ}$ 1, 1971, p. 11-23, ici p. 15.

9. Une étude architecturale et patrimoniale de la prison a été menée en 2016 par des élèves de l'École de Chaillot. Ce travail, associé à des études menées sur d'autres monuments de la ville, a fait l'objet en 2016-2017 d'expositions à Autun et à la Cité de l'architecture et du patrimoine.

10. BENTHAM Jeremy, Panoptique. Mémoire sur un nouveau principe pour construire des maisons d'inspection et nommément des maisons de force [1791], Paris, Mille et une nuits, coll. «La petite collection ", 2002, p. 33.

11. Circulaire du 17 août 1853 disponible via https://criminocorpus.org/fr/ref/25/17040/ [lien valide en septembre 2021].

12. Notes du gardien-chef, 15 septembre 1857, Autun, AD Saône-et-Loire (ci-après AD SL), 4N 28.

13. Courrier d'André Berthier au préfet, 28 septembre 1857, Mâcon, AD SL, 4N 28.

14. Rapport de l'architecte du département, sur le projet par lui dressé, des travaux à faire pour la construction de nouveaux bâtiments destinés à servir de prison pour l'arrondissement d'Autun, 20 août 1847, Mâcon, AD SL, 4N 28.

15. Procès-verbal du conseil d'arrondissement d'Autun, 19 juillet 1858, AD SL, 4N 28.

16. Budget de 1860, département de Saône-et-Loire, arrondissement d'Autun, prison, grosses réparations, AD SL, 4N 28. 
17. Témoignage oral direct de l'ancien propriétaire de la prison, recueilli par André Strasberg, alors conservateur du musée Rolin.

18. Rapport sur l'établissement de treillages et de toits sur les préaux, A. Berthier, 1858.AD SL, $4 \mathrm{~N} 28$.

19. Budget de 1860, département de Saône-et-Loire, arrondissement d'Autun, prison, grosses réparations $\mathrm{AD}$ SL, $4 \mathrm{~N} 28$.

20. 5 juin 1875. Loi sur le régime des prisons départementales disponible via https:// criminocorpus.org/fr/ref/25/17156/ [lien valide en septembre 2021].

21. À ce sujet, consulter CARLIER Christian, «La construction des prisons en France au $\mathrm{XIX}^{\mathrm{e}}$ siècle : de longues hésitations ", Paris, Centre pour les humanités numériques et l'histoire de la justice, 2009, [en ligne] https://criminocorpus.org/fr/ref/25/17367/ [lien valide en septembre 2021].

22. Rapport de l'architecte départemental, 10 janvier 1879, Mâcon, AD SL, 4N 28.

23. Rapport de l'architecte départemental, Mâcon, 10 janvier 1879, AD SL, 4N 28.

24. « Programme architectural, fonctionnel et technique pour la rénovation et l'extension du musée Rolin à Autun », groupement Mikaël Seban, architecte programmiste, juin 2019.

\section{RÉSUMÉS}

La prison circulaire d'Autun, conçue en 1847 par l'architecte André Berthier (1811-1873), est un exemple unique en France de prison panoptique ayant adopté le plan circulaire. Inscrite puis classée monument historique en 2017, elle intègre aujourd'hui un projet muséal qui conduit tout autant à la redécouvrir qu'à en bouleverser son usage. Le lancement des études architecturales en 2020 incite à revenir sur ce projet si fidèle à l'esprit de son époque pour tenter d'en mieux comprendre les réussites et les faiblesses, tant sur le plan architectural que fonctionnel, et explorer quelques projets de modifications dont il a fait l'objet.

Cette prison est une traduction à la fois fidèle et originale des concepts qui prévalent lors de sa conception dans les années 1840, prônant l'enfermement individuel et s'appuyant largement sur l'idée de la prison panoptique développée par Jeremy Bentham (1748-1832). La traduction rigoureuse de ces idées dans sa forme architecturale entrave cependant, dès son ouverture, sa capacité à s'adapter à de nouvelles mesures, tandis que des faiblesses sont rapidement relevées dans le bâtiment. Dans les premières décennies qui suivent son ouverture en 1856, elle fait l'objet de différents projets de réaménagement qui expriment à leur tour les nouvelles préoccupations et mesures correctives jugées nécessaires dans cette maison d'arrêt qui semble n'avoir jamais fonctionné selon les plans de son concepteur ${ }^{1}$.

The circular prison of Autun, designed in 1847 by the architect André Berthier, is a unique example of a panoptic prison that adopted the circular plan in France. Recognised as a national historic monument in 2017, it is now part of a museum project that will lead just as much to its rediscovery as to change its uses. The launch of architectural studies in 2020 has encouraged us to return to this project, in line with the spirit of its time, trying to understand its successes and weaknesses, both architecturally and functionally, and to explore some of the modifications it has undergone.

This prison is both a faithful and original translation of the concepts that prevailed when it was designed in the 1840s, advocating individual confinement and largely based on the idea of the 
panoptic prison developed by J. Bentham. The rigorous translation of these ideas into its architectural form, however, hindered its capacity to adapt to new measures, while weaknesses were quickly identified in the building. In the first decades after its opening in 1856, it was the subject of various redevelopment projects which in turn expressed the new concerns and corrective measures deemed necessary in this prison, which seems never to have functioned perfectly as its designer had intended.

\section{INDEX}

Mots-clés : palais de justice, panoptique, prison, Bentham (Jérémy), utopie

Keywords : courthouse, panoptic, prison, Bentham (Jeremy), utopia

\section{AUTEUR}

\section{AGATHE MATHIAUT-LEGROS}

Ville d'Autun, directrice des Musées et du Patrimoine, conservatrice en chef du musée Rolin agathe.legros@autun.com 